\title{
Structural and functional aspects of arginine decarboxylase from Salmonella typhimurium
}

\section{Geeta Deka ${ }^{1}$, Bharath $\mathrm{SR}^{1}$, Murthy M.R.N ${ }^{1}$, Savithri H.S ${ }^{2}$}

${ }^{1}$ Molecular Biophysics Unit, Indian Institute Of Science, Bangalore, India, ${ }^{2}$ Department of Biochemistry, Indian Institute of Science, Bangalore, India

E-mail: geetadeka@mbu.iisc.ernet.in

Salmonella typhimurium colonizes the human gastro-intestinal tract is due to its ability to withstand high acidic $\mathrm{pH}$ ( $\mathrm{pH}<$ 2.5). Its acid tolerance response (ATR) is due to lysine decarboxylase (LDC) and arginine decarboxylase (ADC) systems, which maintains the internal $\mathrm{pH}$ of the bacterium close to 4. ADC consumes one proton from the cytoplasm while decarboxylating an arginine to agmatine. The arginine-agmatine antiporter (AdiC) exchanges an internal agmatine with an external arginine. Thus, together, ADC and AdiC reduce the proton concentration within the cell. Here, we present the X-ray crystal structure of the inducible StADC (AdiA) at $3.1 \AA$ resolution. The crystal asymmetric unit contains a decamer (MW $800 \mathrm{kDa}$ ) constituted by five homodimers related by a non-crystallographic five-fold axis of symmetry. The structure represents the apo-form of the enzyme while the earlier reported structure of the E. coli enzyme corresponds to the holoform (Andrell et al., 2009). Binding of PLP converts two disordered loops close to the active site into ordered confirmations. These conformational transitions may be important for substrate entry and product release. A large number of acidic residues are found on the surface of StADC. As proposed earlier (Andrell et al., 2009), low pH may neutralize surface charges in homodimers that are catalytically inactive and promote the formation of functionally active decamers. Comparison of StADC with other members of group III decarboxylases shows that these enzymes are likely to follow similar catalytic mechanisms.

(1) Andrell, J., Hicks, MG., Palmer, T., Carpenter, EP., Iwata, S., and Maher, MJ. (2009), Biochemistry 48, 3915-3927.

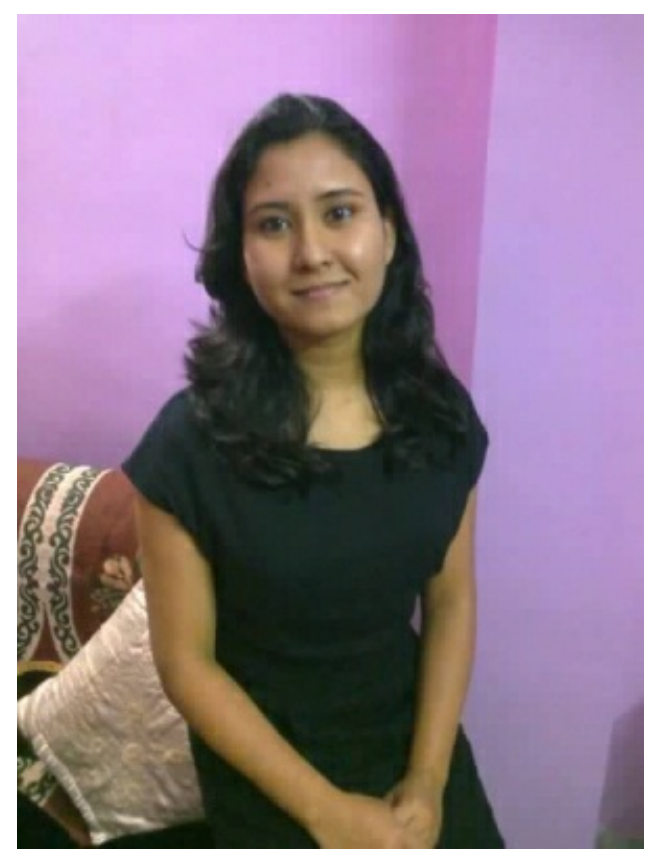

Keywords: Arginine decarboxylase, Crystal structure, PLP 